\title{
Introduction to Profiling: The Process of Reading of Nonverbal Signs
}

\author{
Associate Professor Magor E. KÁDÁR, PhD, habil. \\ Faculty of Political, Administrative and Communication Sciences \\ Babes-Bolyai University Cluj Napoca, Romania \\ E-mail: kadar.magor@fspac.ro
}

\begin{abstract}
The present study debates the process and the difficulties of the nonverbal sign reading. As the perception and decoding the signs are strongly connected to the personality the first aspect is to know establish a proper baseline the subject will be referred to and have a proper selfknowledge to be able classify the signs perceived. The paper focuses on process offers a possible way for reading and understanding followed by the classical face sign classification. Recommended to know it but also to develop a personal reading process and reference line appropriate for the people, culture, environment and circumstances of meeting. The last part will offer a brief list of sources to gather information in any interpersonal process as a start for everyone's own readings.
\end{abstract}

Keywords: Profiling, nonverbal communication, interpersonal psychology, signal reading and interpretation.

"I see it, I deduce it. How do I know that you have been getting yourself very wet lately, and that you have a most clumsy and careless servant girl? (...) It is simplicity itself... My eyes tell me that on the inside of your left shoe, just where the firelight strikes it, the leather is scored by six almost parallel cuts. Obviously they have been caused by someone who has very carelessly scraped round the edges of the sole in order to remove crusted mud from it. Hence, you see, my double deduction that you had been out in vile weather, and that you had a particularly malignant boot-slitting specimen of the London slavery." Conan Doyle: A Scandal in Bohemia 
Nonverbal codes can be divided into two large groups. In the group of representation codes the most developed system is verbal language which is associated with formal characteristics such as the ones referring to the environment of transmission (personal meeting, audio and audio-visual media, etc.) as well as conventions related to their social use. The receiver decodes them by these means. The channel of transmission for presentation codes is the human body. The basic types are behavior codes and the learned ones (e.g. body movement), commercial codes (e.g. clothing) and genetic body codes (e.g. facial structure, hair color). The latter one is the source of information for automatic stereotypes that play an important role in rapid reading and the forming of first impressions.

The codes from these three categories may express four types of indicators:

a. indicators of interpersonal relationship,

b. indicators that reflect social class,

c. indicators of identity,

d. indicators of expression.

Depending on the type of indicators we may define groups of analysis and reference, with which we can decode and interpret the messages sent. ${ }^{1}$

A hybrid field of verbal and nonverbal communication is kinesthetic, which analyses the movements of the body during the creation of speech. In the process of reading the words from the lips it is important to follow the movements of the lips, neck, thorax which accompany speech, but sometimes we interpret a picture or a silent film in a similar way (e.g. determination of sonority), an effort or work process (e.g. rhythmic shouts or their encoding into a work tune) or mass events (e.g. the behavior of sports fans).

Communication of the content is taken over by more developed communication systems which are characteristic to humans, such as writing or language. Therefore, we are going to follow and interpret nonverbal messages with less awareness than verbal language, and this is true both for the communicating person and the receiver. ${ }^{2}$ Since nonverbal messages represent a less controlled way of communication, they reveal certain attitudes or feelings which the communicating individual does not want to show. Nonverbal chatter represents this additional information, among others the situation when a person is telling the truth or lying. ${ }^{3}$ It has a great importance in psychiatric treatment ${ }^{4}$, in the case of compen-

1 See also the work of Michael Argyle.

2 Cultural characteristics which (for example) do not accept the free expression of interpersonal feelings and attitudes in any situation, may also contribute to this.

3 Forgas based on Ekman-Friesen.

4 The idea of reading and interpreting nonverbal signs occurred related to the evolution theories as well, as they are systems of communication older than language, and were initially used in psychiatry and C.I.D. 
sated patients (who do not present symptoms) who try to mask their anxiety and excitement.

During the experiments the observers included their evaluations on the emotional state of the patient in the categories mentioned above, on the basis of the communication sign of the head, body or both. At the end they formulated several conclusions which have later been completed by others as well (see Argyle). Nonverbal messages do not only accompany verbal communication but they convey more effectively attitudes and feelings than verbal communication.

Nonverbal signals influence the receiver deeper than verbal messages and the receiver always has greater confidence in nonverbal messages as they influence his decisions.

Table I. Nonverbal chatters

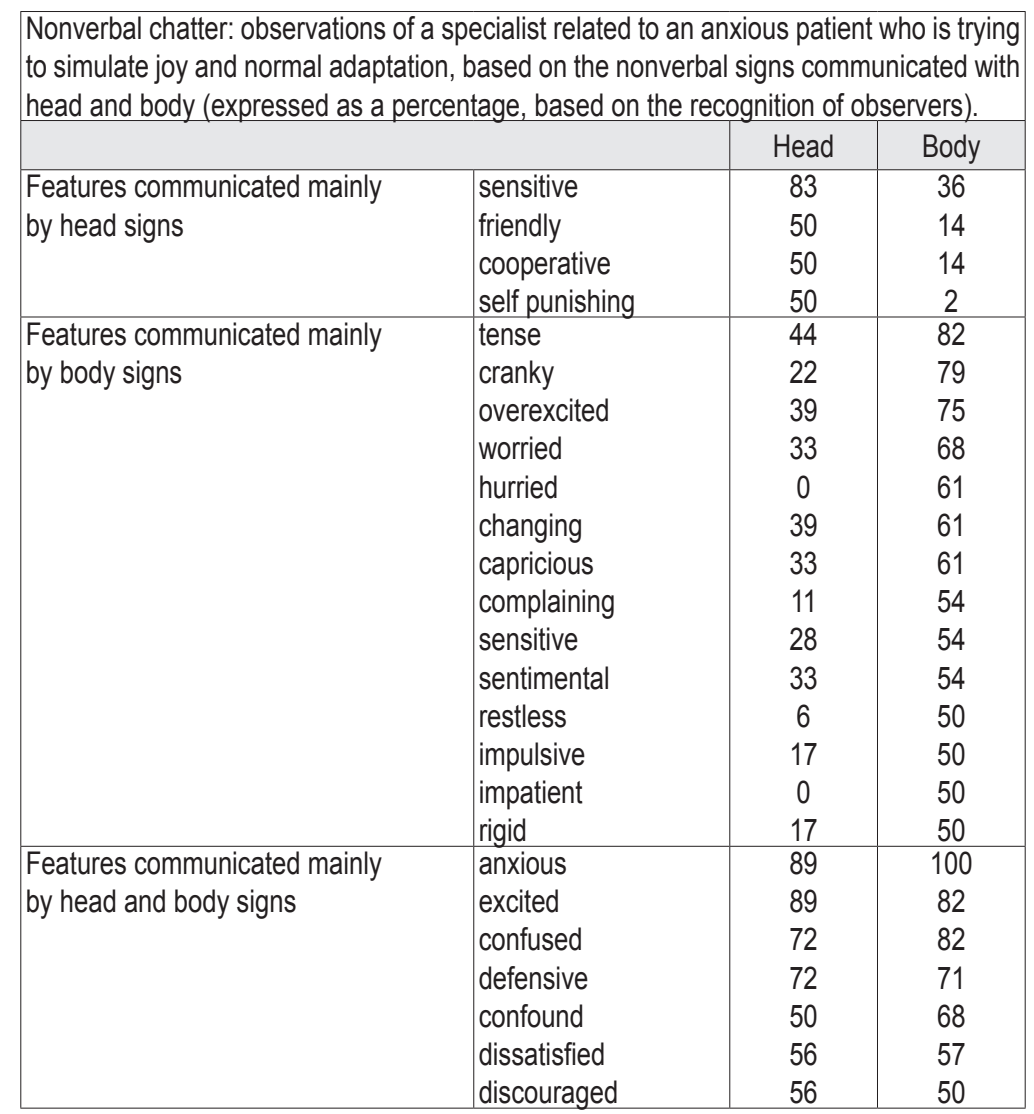

The conclusion is that "language may be used successfully to communicate information related to the world around us and the problems waiting to be solved, while nonverbal messages play an important role in the communication of social values, attitudes, affinity and other personal reactions." (Forgas n.y. 159). 


\section{The difficulties of sign reading}

"If at a poker game you look around and you can't tell who's going to lose, it will be you."

Net folklore

In the recognition of nonverbal signs we may face two problems. The first one is the feeling expressed by the target person while the other one is the receiver's ability to interpret correctly. For the communicating person it may be difficult to express feelings clearly because people rarely react with a single feeling to a certain situation (Forgas n.y. 37-38). People usually react with a mixture of feelings which are expressed by mimics. However, it is not unequivocal that the same impulse (sight, situation, information) will generate the same feeling from different people (e.g. listening to music can relax, raise attention, generate emotion, disagreement, sometimes even disgust or fear).

Basic emotions may be recognized and identified in most cases. But in everyday life situations are more complex and cannot be expressed by pure feelings; on the other hand they are very ephemeral and conscious analysis is difficult without fixation and reproduction. As we are dealing with natural reactions and relationships from the real life, the advantage is that we do not need to limit ourselves exclusively to facial expressions. The reading and interpretation of facial expressions may be supported by information provided by the environment and the situation (possibly experienced by the receiver), the use of multiple nonverbal channels, previous communication and already existing data. "The reader is always requested to keep in mind that these signals are not used in an isolated manner: the message consists of several components." (Forgas n.y. 173).

From the point of view of the receiver the problematic elements were discussed in the previous chapters where the problem was analyzed from different perspectives. Going through the stages of perception, reception and processing, the following problems may occur:

1. Problems related to perception (Kádár 2014):

- distortions generated by the prejudices of the receptor

- distortions generated by cultural differences

- differences between the environment of the manifestation,

- the length of the event.

2. Interpretation filters (Kádár 2014):

- Automatic stereotypes, primary effect,

- relationship, stereotypes, prejudices,

- the ability to assign,

- the ability of individuation and drawing conclusions (Kádár 2015).

3. The interpretation process (Kádár 2014):

- the perception process is not objective, 
- the perceived information is never complete,

- classification is already included into the perception (encoding process),

- types, then categories and schemes are developed during classification,

- schemes are easily transformed into stereotypes,

- stereotypes are activated very quickly.

4. The observing person:

- training, qualification and exercise,

- the rapidity of processing, the ability of storing,

- conscious control of stereotypes and getting rid of them (Kádár 2015),

- living the emotional state and the given situation,

- closeness and feelings towards the target person (Kádár 2015),

- verbal coverage,

- the importance or stake of the observation.

5. Problems related to the environment:

- noise coming from the environment, distance,

- the perceptible channels used by the target person,

- the abundance of data,

- divided concentration in case of multiple target persons.

Besides the communication method, mentality and the processing ability of the target person, environmental conditions (noise, the reaction of the environment, etc.) may represent a problem as well. There are two approaches to this: the first tries to maximize the amount of information received, the more data we have the more accurate the conclusion would be, while the second one tries to reduce the amount of data and avoid the confusion created by the abundance of information. Maximizing information is beneficial in situations where the observer has fewer paths because he tries to identify others, avoiding attributing much importance to the few available signals. In order to perform a sophisticated analysis we need more information and the full observation of certain processes, but these are usually done in groups, and the analyzed material is recorded, so it can be viewed and reviewed. When two persons meet it is sufficient to observe the most relevant channels to avoid confusion created by the abundance of data. Even if in addition to the mainstream information (strong expressions of the clear, dominant status) the well controlled paths will transmit other signs as well, the amount of data is so vast that the observer is not able to receive, divide and interpret them. So he applies the principle of "less is more", and in this spirit there is a minimum amount of required data which helps to create a clear image, the rest of information is excessive and confusing. ${ }^{5}$

5 Reading based on limited data, the analysis of the correctness of the first impression (blink) and spontaneous associations have a special literature - see the references of Gladwell (2005). 


\title{
2. Reading methods
}

\author{
"The one who observes me carefully will know me. \\ Therefore I'm silent, and through silence I communicate more." \\ Illés-Bródy-Koncz: Jelbeszéd (Sign language)
}

The transition from the "soft image" based on impressions and individuation to the "hard image" of descriptions is difficult. The process of becoming aware, during which facts are formulated and attributed to scales and schemes, then their verbal or written formulation involves not only the loss of a certain amount of information but also the loss of those "weak relationships" which are impossible to formulate but which make the perceived things understandable and offer the possibility to "get" a feeling, solution or correct observation spontaneously, almost without any sing. Verbal screening generates obtrusion while associations are caused by semi-conscious operations and background information.

Verbal screening occurs when the perceived signs are recoded into oral or written form. The consciously performed description attenuates the ability of recognition and automatic association which do not require conscious thinking. The left hemisphere of the brain performs the encoding into words while the right one into images, in the case of descriptions the data of visual memory are encoded into words, e.g. describing a face, physical appearance or environment (analysis carried out by Johathan W. Schooler (Gladwell 2005, 98-99). The encoding will not be perfect, of course, as details and (especially) relationships cannot be formulated explicitly. If the encoding is performed, our memory will later turn to the already described stories or features in their quality of rough information, and not to the original image or impression. It is true that impressions fade and are overwritten in time but it is equally true that descriptions, although persistent in time, cannot offer very important details. Therefore the profiler who does the encoding needs exercise and experience to offer a second receptor the chance to evoke the situation exactly.

The complex system of communication signals represents a communication carried out in several ways. Signals arrive concurrently, first through the channels of body language (facial expressions, posture, gestures of hands and feet) but also by vocal and non-verbal ways, which in terms of the analysis may be treated individually but their interpretation is only possible together. Since the focus is primarily on the message, nonverbal ways gain less attention. Some of them (mimic, for instance) are easier to control than the rest. ${ }^{6}$ The prioritization of signals begins with the signals transmitted through less controlled channels, the role of the controlled ways is to confirm them; then the totality of signals may be compared with the formulated content and the opinion of others. Beside priority and the explicit nature of the sig-

6 The use and control of the channels may sometimes depend on the culture, in Japan for instance the diminished mimic is very important. 
nals an important criterion is the harmony of signals, messages may be considered explicit only where multipath signals indicate the same attitude or feeling.

Channel order is followed by analysis from the perspective of the process. The mood of a particular moment matters less but the process of transformation (the point from where we started and the end) is important. During a speech the initial tension or refusal may be followed by opening or even interest, and later on by acceptance and interaction with the interpreter (up to posture reflection), which may turn into boredom, disinterest, and end with the signs of departure or termination of the relationship. At one point, certain communication channels transmit dominant attitude while others may already indicate the following attitude - which will only emphasize during the analysis (direct observation, film, series of pictures).

Similar to the ways of communication we need to establish a priority order among controlled, uncontrolled and semi-controlled indicators. Indicators are explicit signals denoting a feeling or state of mind, their recognition is easy, so their conscious use is highly recommended. Controlled indicators are signals used consciously (e.g. the gesture of presentation, the smile when meeting someone, or the previously practiced gestured at a meeting of public speech), they are usually transmitted consciously, through controllable channels. Semi-controlled signals are partly instinctive and partly fixed by exercise and routine. Their decoding requires some knowledge of the target person, so that style indicators can be distinguished from the less controlled indicators (to separate the conscious signals from the spontaneous ones). Involuntary indicators are signals expressed spontaneously and they are the most valuable in terms of reading and interpretation. In practice, public figures try to limit involuntary indicators and their routes to avoid the gap between these and the message communicated. This way, however, they can neither support nor accompany the message. ${ }^{7}$ The received indicators may be included into a special category of nonverbal communication.

Profiling means, in fact, drawing conclusions which the profiler does not get indirectly but because he became an observer. According to the theory of teleology, signals serve to a better expression of the message and they were born with this purpose. Profiling, the science of observation, was developed based on this idea and it means focusing on the target person, observing and recording signals to the smallest detail and, finally, summarizing the conclusions. The target person is compared with himself and the observations refer to the same person in different environments, thus the person may be compared with himself. The rules

7 During negotiations massive tables are preferred to armchairs, because they partially cover the gestures of the feet and the posture. Lectern is often used for political discourses, the speaker stands behind it, his posture is straight and his hands rest on the lectern. There are only two channels of communication left in such cases: the mimic and voice, which are the easiest channels to control. 
of observing the indicators of behavior may be summarized as follows (see also Collet 2003, 293-294):

1. The accuracy and credibility of the conclusions is directly proportional to the number of indicators available for the observer. Any further information calibrates and therefore validates the conclusion.

2. We shall not jump to conclusions. Until a specific finding is not confirmed by other sources, it only remains a guess.

3. Each person must be related to himself. Observations shall refer to the same person, in similar and in different situations.

At the first meeting the observer cannot know yet which of the signals of the target person are specific, whether they are part of his habits and style (whether they result from education or cultural orientation, or are generated by influences coming from the environment, state of health, etc.). In such cases we have two methods of judging a person's perception:

1. We shall establish a general system of references based on impressions and stereotypes, taking into account both the person and the environment (see also Collet 2003, 298-299). In this case signals can be interpreted as signals transmitted by a person in a similar situation. When the individuation takes place, over a longer period of observation the target person becomes more familiar and based on existing information we may establish a personal profile, and initial signals can be reinterpreted.

2. Through a thorough analysis the observer fills the schemes with content, compiling a profile which, in order to be accurate, is compared with a standard scale or with the profile of the same person prepared by other observers (Forgas ny, ch. 2). Although this method requires more time, it provides a more precise result, responding more accurately to professional dilemmas related to judgments about a person's perception.

The literature contains several systems of references and measurement or previously outlined schemes depending on the point of view from which the target person or smaller populations are observed. ${ }^{8} \mathrm{~A}$ very common method of analysis is the Big Five competence test which measures the characteristics of personality based on five features (Gladwell 2005): ${ }^{9}$

1. Extroversion (exteriorized). Does he like the company of others or is he more reserved? Does he enjoy having fun or is he retained?

8 Analyses are usually psychiatric evaluations but they started to be frequently used in evaluations for the labour market, marketing policies, etc.

9 Proficiency test can be successfully used for the analysis of spaces and their use, and to data provided by close friends of the target person. Its disadvantage is that it is a written test which often limits the expression the person who completes it. 
2. Friendly character. Does he trust people or is he suspicious? Is he friendly or refuses cooperation?

3. Conscientious. Does he like order or is he untidy? Is he able to discipline himself, does he have self-control or he has no will?

4. Sentimental stability. Is he worried or calm? Does he feel safe or is he insecure?

5. Open towards novelties. Does he have a creative imagination or he prefers facts? What is more characteristic to him: individualism or conformation?

Profilers are always aware that the predator is at the same time prey, or in other words: the environment acts on the observer as well. The observer perceives not only the environment, gathers information and draws the conclusions but is an integral part of social situations and processes; he is also observed and conclusions concerning him are formulated too. This often focuses more attention on the overall image and non-verbal communication and this sensitivity influences the more thorough reading of others. Awareness of someone's own communication and behavior ensures the development of a certain routine, sparing the observer of different manipulative processes used during the observation. The conclusions, however, make the observer more responsive to his environment and to social problems, making him undoubtedly a connoisseur of these situations.

The conscious attention of the observer on his own communication often generates attitude problems, in other words his negative traits of character are highlighted. The most common such situations are:

- the observer thinks he can easily read others and becomes conceited and shallow,

- he considers himself more important than others because he is in possession of a certain knowledge or skills that others do not have, because he stops to develop,

- believes that he cannot be manipulated, therefore he is not careful to other people's such attempts, so by lacking a critical attitude he is easier to be influenced.

Reading and proper preparation of a profile requires constant self-education and self-awareness, situational awareness and the feedback of the professional environment without that the work of reading cannot be performed successfully.

Regarding the process of sign reading as a whole, we may say that:

1. Signs should be codified in written or oral signs because this way the information is recorded and can be evoked, but sometimes the clarity of the impressions and relationships is influenced.

2. Signals transmitted through different channels may only be interpreted as a whole. We need to take into account the signals of each channel and we may observe them separately, but the interpretation shall refer to their totality. 
3. The core message should arrive through several channels at the same time, but only the message conveyed by harmonizing signs is relevant. The transmitted feelings and attitudes change over time, so the assessment of the process represents more than momentary signs.

4. The least controlled way is the most plausible one, but it has to be accompanied by other signals, transmitted in other ways.

5. The "overcommunicative" nonverbal communication or nonverbal chatter may be regulated by limiting the pathways. Interpretation will have sufficient sources even this way.

6. If we have enough time, the observed person shall be compared with himself but we may also establish comparison schemes and systems.

7. The environment acts on the observer as well. The observer is observed and analyzed by his environment, so he needs to pay attention to his projected image and nonverbal communication.

This chapter is only a guide to start reading. Comparison schemes and the keys of reading codes are needed and from the described processes we may choose the ones that fit the situation and purpose. The results should always be checked by comparing them with other observations or older conclusions.

\section{Reading the facial expression}

"Human face is an enigma but for those who have the proper key to it, is an open book."

Mór Jókai

Reflecting the most nuanced feelings, facial expression is probably the most communicative way which is considered the most easy to read because humans can express basic feelings from a very early age and it can be read consciously or instinctively. Of all nonverbal channels facial expression is one that has a general value, its signals can be recognized regardless of race or culture, they overcome cultural differences and those related to education (the alleged cause may be that some of the mimic is encoded genetically while gestures are fixed on a cultural background).

The movement of facial muscles can be divided into mimical units which may help to identify more complex expressions (Ekman-Friesen 2003; Birkenbihl 2007). The description, the possible combinations and their interpretation can be found in the 500-page book written by Ekman-Friesen, called FACS (Facial Action Coding System). ${ }^{10}$ Their professional activity refers to the recognition and analysis of

10 Ekman, Paul - Friesen, Wallace V.: Facial Action Coding System, 1 \& 2. Human Interaction Laboratory Dept. Of Psychiatry, University of California, San Francisco (Gladwell 2005). 
fundamental feelings that define the following sentimental dimensions: surprise, fear, disgust, anger, happiness, sadness (Ekman-Friesen 2003, 34-128). The mimic signs of these basic emotions are:

1. Surprise:

- eyebrows are raised and arched,

- the skin under the eyebrows is stretched,

- horizontal wrinkles appear on the forehead,

- eyelids are open; the upper eyelid raises, they white of the eye is visible above the iris; the inferior eyelid is tense, we may often see the white of the eye here too,

- the jaw is lowered („his jaw drops”), the lips open but they do not have a tense tonus and they do not stretch over the teeth.

2. Fear:

- forehead wrinkles are grouped towards the centre and they do not stretch along the entire forehead,

- eyebrows and raised and frowned,

- the upper eyelid is risen, the white of the eye appears above the iris and the lower eyelid is raised and tense,

- the mouth opens, lips are often tense and pulled back or are strained to the maximum and pulled back

- the mouth opens, lips are often tense and pulled back or are strained to the maximum and pulled back.

3. Disgust/antipathy:

- the upper lip rises,

- the lower lip either rises to the upper lip or is lowered and pushed in front,

- the nose wrinkles,

- the cheeks rise,

- wrinkles appear under the lower eyelids, eyelid are slightly risen, without being tense,

- the eyebrows are lowered and push the upper eyelid downwards.

4. Anger/rage:

- drooping, frowning eyebrows,

- vertical wrinkles appear between the two eyebrows,

- the lower eyelid is tense, sometimes it is raised

- the upper eyelid is tense and sometimes is risen, depending on the movement of the eyebrows,

- eyes are fixed and create the impression of staring, sometimes bulging,

- lips may have two states: they can be pressed tightly, the corner of the mouth is in a straight line with the mouth or is bent down; open, rough like when shouting 
- nostrils dilate but this is not a fundamental sign (it sometimes may indicate sadness)

- the indication of anger is explicit only when it is transmitted by the entire mimic of the face.

5. Happiness:

- the corner of the lip is lowered and pulled to the back,

- the mouth may be closed or slightly open, teeth may show,

- a wrinkle stretches from the nostrils towards the corner of the mouth,

- the cheek rise,

- wrinkles appear under the lower eyelid which may be risen or not but it is not tense,

- wrinkles appear in the outer corners of the eye ("crow's-feet");

6. Sadness:

- the inner side of the eyebrows rises,

- the skin under the eyebrows has a triangle shape and one of the corners points upwards,

- the interior corner of the upper eyelid rises,

- the corners of the mouth are lowered and lips may tremble

Although they may be read immediately, basic expressions are quite rare and only occur for a short period of time. Their trigger is not always relevant, from the emotional point of view people respond differently to the same impulse. Mixed feelings are common but when we separate and interpret them, we need to take into account the entire body language. This proves the fundamental principle that it is not enough to read one channel, even if it is as eloquent as mimic. ${ }^{11}$ Mixed feelings (e.g. the feelings caused by multiple simultaneous impulses, sending replacement signals based on self-control, etc.) offer at the same time the possibility of hiding; the number of expressed feelings can be increase deliberately and thus separation and interpretation becomes more difficult.

Seeing the reactions of the communicating person, the facial muscles of the observer will automatically reflect those seen. This phenomenon is called imitation empathy that facilitates interpretation if the receiver is aware of the adopted feelings. In addition, there is the role of mimic in generating feelings: the occurring of the feeling and facial expressions are directly connected through nervous pathways, and the facial expression generated by the movement of the proper muscles

11 Experts and specialized software to analyse facial consider that the face is fully representative because if it is separated into areas and micro-expressions, it can fully express feelings. The other channels are employed only because micro-expressions are not always visible to the naked eye and the observer does not always have access to recording instruments. 
will cause the appropriate feeling. The adopted facial expression evokes a particular state of mind to which the appropriate physiological symptoms are added (increased body temperature or heart beat, sweaty hands, etc.). Therefore, mimic is not only a byproduct of feeling or an associated phenomenon; the process works the other way round, mimic has the same role in the generation of emotions. ${ }^{12}$

Asian medicine uses the method of emotional reaction for a long time. This is a two-way process, from the fixed mimic features we can read a person's character while changes indicate the presence of different diseases; "there was always a clear relationship between physiognomy and human features." (Ohashi 2002, 40). The adopted mimic helps healing, influencing not only the change in mood and the attitude towards illness but it also generates physiological symptoms which support healing.

The "reading of the body" focuses on the face, divided into three sections, each having assigned a part of human nature (Ohashi 2002):

1. the forehead mirrors the intellectual character,

2. the space between the eyes and mouth reflects the emotional character,

3. the area situated under the nose, around the mouth and chin reflect the power of will.

The principle of "totality is made of details" or "macro is formed of micro" applies when the reading of the face and body is done: the entire body may be projected on the body or language, and we may read the changes.

\section{Reading body language}

"If someone is trying to piss you off, it takes 42 muscles to frown but only 4 to smack him."

Net folklore

Body language represents the transmission of message on multiple levels, including kinesics (head posture, gestures of the arms and legs), posture and body position (posture communication) and the space regulating behavior. During the analysis we shall take into account the complexity and harmony of all these ways. Messages can be considered explicit if the gestures of the face, hands, arms and legs and the posture of the body reflect the same content. Then they should be compared to facial expressions, voice and other nonverbal ways.

Although facial expression conveys the most feelings, its control is fairly strong. Therefore we must also take into account body language signals which can complement or refute the messages conveyed by facial expression or mimic. Reading starts at mimic and is followed by signals related to the head and face, the gestures of the arms and legs, then the posture of the head and body, and the use of space.

12 Experiments done by Ekman, Friesen and Levenson (Gladwell 2005, Forgas n.y.) 
There are several classifications but in the case of reading the first step is to make the separation depending on the value of the content. There are attitudes, feelings, signs expressing the state in a given moment, and there are additional or transitory signals, idletimes which fill the time between two gestures replacing something (but not very clear, what), gestures of embarrassment, etc.

Signals carrying an increased content value can be classified in many ways, almost every author in the literature developed his own classification. At first glance one may note the open character (similar to the first general aspect of the writing in graphology). Open gestures generate confidence but they also indicate attention and self confidence. Closed gestures indicate anxiety, rejection, dislike and a possible passivity. A vast literature helps us reading the indicators, the selection criterion is whether the signals can be interpreted isolated, independent of the context (which excludes different possibilities and could be interpreted rather wrong than right), or signals explained in relation to other sources or environmental conditions. ${ }^{13}$ For example in most works the rubbing of the lower part of the nose with the index finger is considered a gesture of covering the mouth: the individual lies and covers his mouth. This may be a solution, perhaps the most common, but considering other signals and the environment the list can be completed. Let us talk about a man at the beginning of spring:

- covers the mouth for not telling the truth,

- covers the mouth to avoid saying a particular phrase (which would put him into a dangerous situation)

- the expression of a stressed state, it indicates confusion (the group of facial touches)

- it masks a yawn (the strain of the frontal part of the neck proves this)

- surprise; he would cover his mouth but this is a very feminine gesture, therefore he tries to control himself

- a gesture of assessment, especially when the thumb touches the skin under the mouth,

- calming (if it is a repetitive gesture, like fondling)

- tic (if it is often repeated in the same form)

- feels that he'd need to blow his nose (the finger is stuck to the nose, the movement is more determined)

- the nose is irritated (e.g. due to a cold)

- the nose was irritated and, although the irritation cannot be seen, the gesture is repeated

- the touch is pleasant (in the brain the nerve endings of the nostrils are situated next to the erogenous zones).

13 We recommend the research of Ekman-Friesen, and for reading the volumes written by Peter Collet, Vera Birkenbihl and Pease-Pease. 
The above list refers to a single gesture which can be interpreted only in relationship with signals transmitted by other means and the information related to the environment. Touching the face can express anxiety ${ }^{14}$ (which is the most common way of expression), but the observation should be supported by the environment, the other signals or by the content of the verbal communication.

Signs carrying a low content value are auxiliary actions (groping objects, clothing, repetitive gestures, touching the face and body, tics) that indicate anxiety. Movements can be insecure, the target person may have changed his mind (another gesture has become a priority in the meantime, so a process is restrained because he is afraid of the end) but they could be gestures filling the time or space (these are more common in the case of children).

Transfer objects like cigarettes, coffee cup, glasses may become important during the observation of body language because they may become the object of a series of replacement gestures amplifying the message. Body language is also manifested during group relations, expressing the acceptance, approval or rejection of a person.

The process of reading nonverbal communication (perception, interpretation, conclusion) may be totaled but in the case of a takeover we should be just as cautious as in the case of isolated gestures. Our fundamental conclusions are the following (see also Birkenbihl 2007):

1. The communication of content consists of data. Nonverbal communication, on the other hand, consists of "data referring to data".

2. Complementary information and those referring to the content may be in harmony or not. But harmony is convincing.

3. When reading, nonverbal signals must first be controlled and not "understood" from the very first reading.

4. One not aware of his own nonverbal signs will not be able to recognize the nonverbal signs of the others.

5. The more someone is able to reveal his sentimental world, the more emphatic he will become and able to correctly interpret the signals of others and will be able to identify with other people's feelings.

6. A signal on its own does not mean anything.

7. The sudden change of body posture is usually generated by a sudden change of behaviour.

8. The mimic signs of the three areas of the face shall always be interpreted together.

9. The more a person is overwhelmed by feelings the more vivid his gestures become.

14 On anxiety and security seeking gestures see Collet 2003. 
10.The one who ignores others' intimate zone will ignore their personality as well.

11. The lack of harmony (incongruence) between different signals indicates the existence of a communication disorder but without revealing its cause.

12. The more natural someone behaves the more improbable is that we'd discover a lack of harmony or seizure in the projected image of this person.

\section{Sources of information}

"We can read each other's minds effortlessly and automatically because the information on feelings and social relationships are always in sight, reflecting on people's faces. (...) Faces always show enough information to manage with "mind reading" in our everyday life. "

Malcom Gladwell

We may employ several methods of collecting information but in each case we shall take into account the distortions, inaccuracies of perception, perceptual differences which distort the reading or make it imperfect. These can be used together but in most cases not all communication channels are available at the same time (because the target person is not engaged in the observation) so we may use only a few of them. However, based on the principle of "a part from the whole" within the system theory, ${ }_{15}^{15}$ several methods are sufficient to create a whole image, and the use of new methods would not generate a significant amount of new information.

1. Using psychological tests (scales, questionnaires).

2. Interviewing the target person.

3. Observing the target person. Relating him to his own self or to general schemes (movement, social interactions).

4. References collected from people who know the target person.

5. Analysis of the (real and virtual) environment in which the target person lives.

6. The indirect expressions and self projection of the target person (his curriculum vitae, website, profile and activities within the social media).

Regarding the accuracy of the perception of a person there are many researches and confirmations. The methods of research may be the following (Forgas n.y., based on Cline 1964):

1. presentation methods of the target person:

a. personal (meeting or personal talk),

b. personal (observing from behind a mirror wall),

c. video recording,

15 The principle of holographic reproduction refers to a system in which each particle is directly or indirectly related with the other particles up to a level, each particle is able to reproduce the whole in a given moment. 
d. film,

e. photograph,

f. voice recording,

g. number of test points or attitude points obtained from a standardized object,

h. personal documents (letters, drawings, curriculum vitae),

i. references to the past experiences of the observers;

2. ways of gathering judgments on the perception of people:

a. evaluation on bipolar scales,

b. projecting professional judgment (medical, psychiatric) about the target person,

c. projecting the target person's behavior in a standardized test,

d. evaluation of the target person by a lists of characteristics,

e. the categorization of the target person in comparison with other persons,

f. free description of the target person,

g. decisions concerning the target person (e.g. employment, qualifications, etc.);

3. criteria used for the assessment of the accuracy of judgments:

a. the results of the target person in psychological tests,

b. information obtained from the target person (e.g. self-evaluation),

c. information obtained from colleagues who know the target person (e.g. friends),

d. judgments of colleagues and superiors,

e. explicit information or demographic data,

f. directly observed characteristics or behaviors.

For the observation of the target person we may use the traditional ways of research methodology of which we would emphasize the methods and tools used in criminology:

1. reanalyzing documents,

2. interview: profound interview (soft and structured), questionnaire (completed by the target person or the operator),

3. observation: neutral or participative,

4. experiment.

\section{References}

1. Argyle, M. (1976). The Psychology of Interpersonal Behaviour. Penguin Books.

2. Argyle, M. (1976). The Social Psychology of Work. Penguin Books.

3. Birkenbihl, V. (2007). Signale des Körpers. mvgVerlag, Redline GmbH, Heilderberg, Germany, $3^{\text {rd }}$ edition.

4. Collett, P. (2003). The Book of Tells. How to Read People's Minds from Their Actions by the Big Brother resident psychologist. Transworth Publishers / The Random House Group, London. 
5. Ekman, P., W. V. Friesen. (2003). Unmasking the Face. A Guide to Recognizing Emotions From Facial Expressions. Malor Books, Cambridge, MA, 2003.

6. Ekman, P. (2001). Telling Lies: Clues to Deceit in the Marketplace, Politics, and Marriage. W. W. Norton \& Company.

7. Forgas, J. P. (1996). Interpersonal Behaviour. The Psycology of Social Interaction. Pergamon Books, Oxford.

8. Gladwell, M. (2005). Blink: The Power of Thinking without Thinking. Back Bay Books.

9. Kádár M. (2015). Building Personal Image by the Primary Effect. Creating and Managing the First Impression of a Person. In Balaban, Delia Cristina, Hosu, Ioan, Mucundorfeanu, Meda (eds.). PR Trend. Developement and Trends in Communicatiuon. AMAK Akademie für multimediale Ausbildung und Kommunikation, Germany.

10. Kádár M. (2014). Psychological Background of The Reading of Nonverbal Signs. Introduction to Profiling. In Mucundorfeanu, Meda, Balaban, Delia, Hosu, Ioan (eds.). PR Trend. New Media: Challenges and Perspectives. AMAK Akademie für multimediale Ausbildung und Kommunikation, Germany.

11. Kádár M. (2014). Nonverbal Communication. Channels, Signs, Sign Reading. LAP Lambert Academic Publishing, Saarbüchen, Germany.

12. Ohashi, W., Monte, T. (1991). Reading the Body. Ohashi's Book of Oriental Diagnosis. Arkana Books, USA. 